\title{
Laser-Generated Intense Planck Radiation
}

\section{R. Sigel, Garching}

\author{
(Max-Planck-Institut für Quantenoptik)
}

The physics of radiation in thermal known since Max Planck's discovery of the so-called law of black-body or, as we shall call it, Planck radiation at the beginning of this century. Until recently, it has not been possible to generate Planck radiation with temperatures exceeding $10^{4}-10^{5} \mathrm{~K}$ in the laboratory, because of the strong increase in the radiant energy flux with temperature. From the StefanBoltzmann law one finds a flux of $6 \times 10^{12} \mathrm{~W} / \mathrm{cm}^{2}$ for a temperature of $10^{6}$ $\mathrm{K}$, increasing with the fourth power of the temperature. The losses which arise circulate are so high that the radiation field cannot be maintained by ordinary power sources.

The situation has changed in recent years, however, with the advent of modern pulsed power generators, such as pulsed lasers and particle beams. These sources offer the possibility of generating radiation fields with temperatures of up to about $5 \times 10^{6} \mathrm{~K}$ in the laboratory. This possibility is of great scientific and technical interest, e.g. for the generation of an intense soft $X$-ray radiation source, the laboratory study of phenomena of radiation hydrodynamics, the investigation of matter in a new range of high pressures, and the production of energy by thermonuclear fusion.

\section{The Basic Scheme}

The method of generating intense Planck radiation by a laboratory source is shown in Fig. 1. An external source delivers energy into a mm-size cavity enclosed by a solid wall. With a laser source one, or preferably several small holes in the wall are required for transmitting the beam whereas a particle equilibrium with matter has been well when such enormous fluxes of radiation

beam can penetrate a wall that is thin yet still thick enough to contain the thermal radiation in the cavity. The rapid deposition of energy in the cavity is supposed to heat the inner wall to a high temperature and to generate intense Planck radiation in equilibrium with the wall. For the applications envisaged, it is important that at these elevated temperatures the radiative exchange of energy between different wall elements is so effective, that very uniform conditions are established in the cavity even if the initial wall irradiation by the source is not uniform. Furthermore, because photons propagate with the speed of light, the radiation field may be formed in the empty cavity before it fills with evaporated, hot plasma from the wall.

Crucial to the success of this concept is the confinement of the radiation by the cavity. In contrast to infrared or visible light, no reflecting walls exist for the soft $X$-rays that dominate the Planck distribution at the temperatures under consideration. The photon flux, incident on the wall from the interior of the cavity, is completely absorbed, and the radiation field can exist only in equilibrium with a hot layer on the inner surface of the cavity, re-emitting the incident energy. Consequently, even though the wall material forces the radiation to diffuse, thus reducing the outward energy flux and, in a sense, confining it, a fraction of the available energy is used for simply heating material. If this energy loss is too large, the desired intensity and uniformity may not be achieved.

We note that a somewhat similar situation exists in the interior of the Sun, where the peripheral layers confine the intense radiation field in its centre. Unlike the static situation in the Sun,

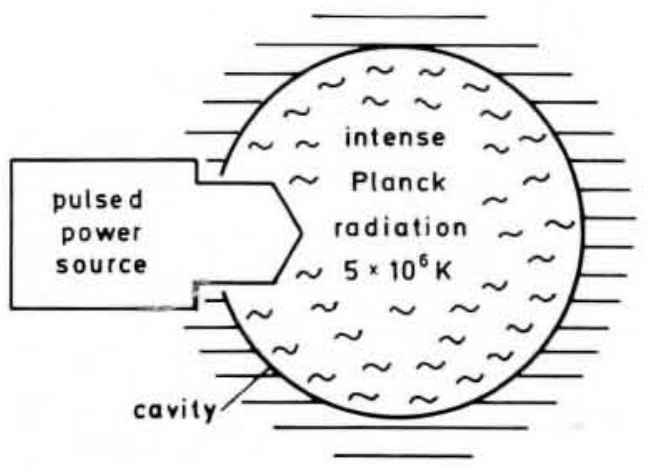

Fig. 1 - Generation of intense Planck radiation in a cavity heated by a pulsed power source. An optimistic estimate of the temperature attainable with existing lasers yields $5 \times 10^{6} \mathrm{~K}$, corresponding to a radiant energy flux of $\sigma T^{4}=4 x$ $10^{15} \mathrm{~W} / \mathrm{cm}^{2}$ and a maximum of the Planck spectrum at $\sim 6 \AA$. however, we are dealing here with a problem which is extremely time-dependent.

\section{The Confinement of Equilibrium Radia-} tion

In order to predict the temperature in the cavity, the radiative transfer problem 1) into the wall has to be solved. The solution has not only to take into account the unavoidable expansion of the heated material (see inset of Fig. 2), but in principle also the thermalization process of the source radiation. In this general form the problem is exceedingly difficult, if not unsolvable.

In a situation where we seek to generate Planck radiation in equilibrium with the wall, it is natural to start by assuming local thermodynamic equilibrium (LTE) between radiation and matter. In the LTE approximation, the real source is replaced by an ideal source, i.e. a source of heat; its physical nature and the thermalization process can thus be ignored. If we postulate in addition that the hot, multiply ionized wall material possesses the maximum possible opacity for thermal radiation (given by a theorem of Bernstein and Dyson) we are specifying conditions which give the highest temperature we can hope to achieve in the cavity.

The major aspects of radiation confinement can be studied in a planar "cavity" consisting of two walls of infinite extent. The LTE assumption reduces the mathematical difficulties to such an extent that the scaling laws for the temperature can be obtained immediately by dimensional analysis from the governing parameters of the problem. These are found merely by inspection of the hydrodynamic equations, which include motion and radiative heat conduction, and the boundary conditions of the problem ${ }^{2}$ ). The result is shown graphically in Fig. 2. The diagram is for a high-Z material (gold) for which the opacity is expected to be closest to the maximum possible.

In Fig. 2 it is assumed that the wall is continuously irradiated with a source flux $S_{s}$. For a short initial period, whose duration depends on $S_{s}$, the radiation mean free path $I_{R}$ is larger than the thickness $I_{T}$ of the heated wall layer and hence radiation and matter are far out of 


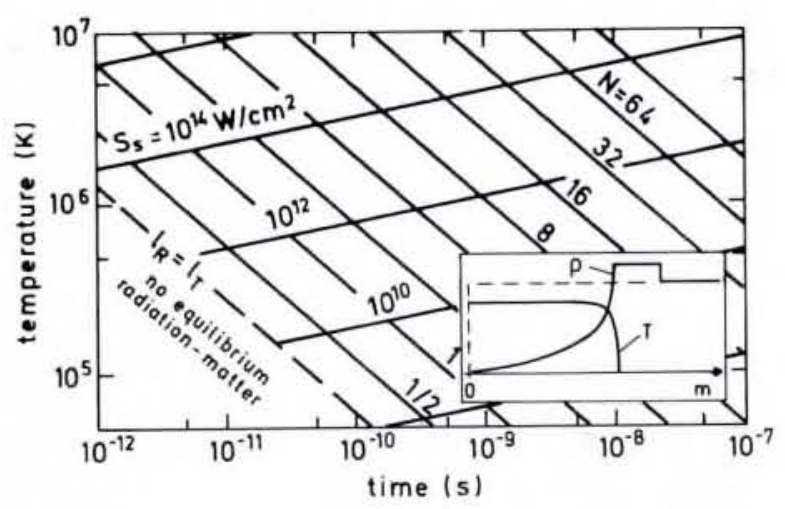

Fig 2 - Increase of the temperature with time if a constant source flux $S_{s}$ is directed against the inner wall of a cavity. $N$ is the quality factor for radiation confinement. The diagram is for an ideal source and maximum opacity. The inset shows the density and temperature profile of the wall before and after the onset of irradiation ( $m$ is the mass coordinate). equilibrium. However, it is expected that to the right of the border line $I_{\mathrm{R}}=I_{\mathrm{T}}$ the LTE assumption becomes gradually valid. Figure 2 shows that the temperature rises with time. This can be understood by realizing that the radiation field in the cavity is in quasistationary balance between the gain of energy from the source and the loss of energy into the wall (transit time effects of the radiation across the cavity are negligible). Because the radiation escaping into the wall has to diffuse through an increasing mass of hot material, the loss decreases with time and the temperature can rise.

Also seen in Fig. 2 are lines of constant $N$, defined as the circulating radiant energy flux divided by the source flux, i.e. $\mathrm{N}=\sigma T^{4} / \mathrm{S}_{\mathrm{s}}$. This quantity is none other than the quality factor of the cavity for incoherent radiation. The symbol $N$ (rather than $Q$ ) was chosen because the quality factor is numerically equal to the number of re-emissions of the source power in the cavity before it is lost into the wall.

Figure 2 shows that $N$ becomes larger than one as the temperature rises along a line of constant $S_{s}$ owing to the confinement effect of the highly re-emitting walls. It is this phenomenon that creates the main interest in cavity heating. It means that a cavity can not only transform the source radiation into soft $X$ rays, but also generate an $N$-fold flux enhancement. The converted radiation that is produced can be radiated through a small hole or absorbed in a small nonre-emitting object with great efficiency. For pulse lasers, it is perhaps most important that multiple re-emissions will smooth out unavoidable irregularities in the initial irradiation so creating uniform conditions in the cavity. This may be of great importance in certain applications as a means of overcoming the fundamental difficulty of obtaining uniform energy deposition by direct irradiation owing to the coherency of laser light.

In a real cavity the heating cannot go on for ever as suggested by Fig. 2. Hot plasma from the wall will fill the cavity and when the plasma frequency exceeds the laser frequency, the laser light can no longer penetrate. Filling can be retarded by choosing a larger cavity, but the surface area is then increased and the laser has to deliver a higher total power. The attainment of high temperatures becomes thus a matter of the power of the laser. We have estimated ${ }^{2}$ ) that in a cavity of $2 \mathrm{~mm}$ radius, irradiated at $10^{14} \mathrm{~W} / \mathrm{cm}^{2}$, heating will self-terminate after about $2 \mathrm{~ns}$. By then a temperature of $5 \times 10^{6} \mathrm{~K}$ with $\mathrm{N} \cong 30$ re-emissions would have been reached and the laser energy delivered into the cavity would have totalled $10^{5} \mathrm{~J}$. Lasers designed to deliver such powerful pulses do now exist ${ }^{3}$ ).

\section{Experiments with Lasers}

Cavity heating experiments have been reported by the Institute for Laser Engineering (ILE), Osaka University ${ }^{4}$ ), and the author's institution (MPQ) ${ }^{5}$ ). Both glass (ILE) and iodine (MPQ) pulse lasers have been used.

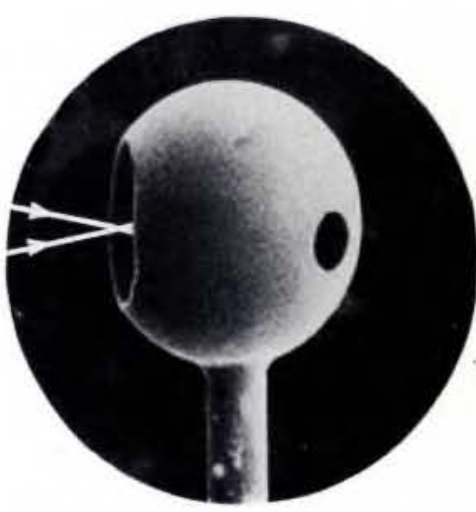

Fig. 3 - Spherical gold cavity (diameter $280 \mu \mathrm{m}$, wall thickness $2 \mu \mathrm{m} /$ with an entrance hole for the laser beam and a diagnostic hole (manufactured by Fa. Dr. Johannes Heidenhain, $D-8225$ Traunreut).

At MPQ, we irradiate very small cavities $(0.25-1 \mathrm{~mm}$ diameter) in order to compensate for the limited laser energy available ( $\leq 100 \mathrm{~J}$ ). The cavities are spherical and made from gold (see Fig. 3). They have an entrance hole for the laser beam and a smaller diagnostic hole which allows us to measure the radiant energy flux and the spectrum of the radiation in the cavity. Recent progress in soft $X$-ray instrumentation has meant that such measurements can be made with the required spectral, temporal, and spatial resolution.

The spectrum is measured absolutely for wavelengths $\geq 5-10 \AA$ using freestanding transmission gratings, made from gold and carrying presently 1000 bars $/ \mathrm{mm}$. The gratings can be integrated into small pinholes for simultaneous

\section{The Pennsylvania State University \\ Experimental Condensed Matter Physics}

The Department of Physics is seeking candidates for tenure track positions in experimental condensed matter physics. Ongoing activities in the department include surface physics, low temperature physics, materials, phase transitions, optical properties and fieldion microscopy. The department wishes to strengthen and complement these research efforts. Salary and start-up funds are competitive.

Candidates should have a Ph.D. in Physics, some postdoctoral experience, and an established record of research accomplishments. A desire and aptitude for teaching of undergraduates and graduate students is essential. Send applications, including a curriculum vitae and names of at least four references to:

Professor Gerald A. Smith, Head, Department of Physics.

Box N.

The Pennsylvania State University, University Park, PA 16802 by 1 January. 1987, or until a suitable pool of applicants is identified.

An affirmative action/equal opportunity employer 

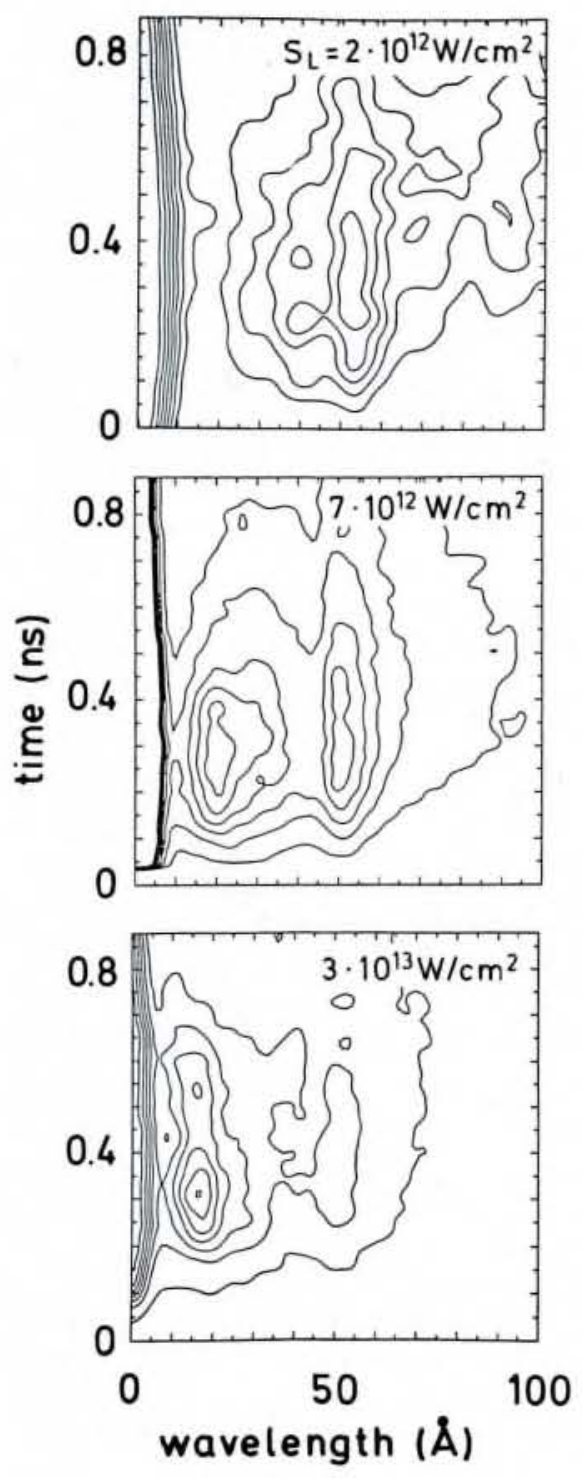

Fig. 4 - Time-resolved soft $X$-ray spectra (iso-intensity plots) from laser heated cavities recorded by an $X$-ray streak camera. Parameter is the average absorbed laser flux $S_{L}$. Along the time axis one half of the zero order is seen. Laser pulse duration was 0.3 ns.

spatial resolution transverse to the dispersion direction. The detector is absolutely calibrated, gelatine-free film. Temporal resolution is achieved with a new soft X-ray streak camera. In the most comprehensive measurements made with this, temporal and spectral resolution are obtained by projecting the $X$-ray spectrum onto the streak slit of the camera. In this way the time-history of the temperature in the cavity can be obtained with about 20 ps resolution.

We present in Fig. 4 time-resolved spectral measurements ${ }^{5}$ ) made at MPO in cavity irradiation experiments at a laser wavelength of $1.3 \mu \mathrm{m}$ for different values of the average laser flux $S_{L}$ absorbed on the inner wall of the cavity. This last is derived from the measured laser energy absorbed, the area of the inner surface of the cavity and the laser pulse duration $(0.3 \mathrm{~ns})$. In all three cases a heating phase, determined by the risetime of the laser pulse, and a cooling phase extending somewhat beyond the laser pulse duration may be distinguished. For the lowest flux $\left(2 \times 10^{12} \mathrm{~W} / \mathrm{cm}^{2}\right)$ the $X$-ray emission occurs preferentially around $50 \AA$, for the intermediate flux $\left(7 \times 10^{12} \mathrm{~W} / \mathrm{cm}^{2}\right)$ around $50 \AA$ and $20 \AA$ and for the highest flux $\left(3 \times 10^{13} \mathrm{~W} / \mathrm{cm}^{2}\right)$ mostly at about $20 \AA$. This shift is not unexpected and corresponds to the shift in the emission of a Planck radiator with increasing temperature.

In these experiments the flux was increased by decreasing the cavity size and using a constant laser energy and pulse duration. Simultaneous measurements of the laser light reflected by the cavity showed that with the smallest cavities (250-280 $\mu \mathrm{m}$ diameter) we had reached the limit for energy acceptance set by plasma filling. For an absorbed laser energy of $20 \mathrm{~J}$ we measured a radiant energy flux in the cavity corresponding to a brightness temperature of $1.3 \times 10^{6} \mathrm{~K}$. A considerably higher brightness temperature of $2.2 \times 10^{6} \mathrm{~K}$ has recently been achieved in joint experiments with ILE, using larger cavities and more energetic laser pulses ${ }^{6}$ ).

A likely reason for the observed structure in the experimental spectra is incomplete equilibrium in the cavity not unexpected with such low energy laser pulses and cavities consisting of a real material whose opacity is less than the theoretical maximum. To interpret the spectra, more detailed calculations which take the spectral opacity of the actual wall material into account have to be done.

For the calculations presented here it is assumed that the density and temperature profile of the hot wall material are given by the LTE solution of the radiative transfer problem, the ablative heat wave ${ }^{2}$ ). Spectra are obtained by solving the radiation transfer equation for these profiles on the basis of spectral opacities for gold, calculated in a hydrogenic average ion model ${ }^{5}$ ). Figure 5 shows the Planckian spectrum corresponding to the LTE prediction for the wall temperature and the calculated spectrum radiated by the hot wall material.

The calculations reproduce well the observed maxima (attributed to transitions in the $\mathrm{N}$ - and $\mathrm{O}$-shell of multiply ionized gold) as well as their shift and relative intensity. Thus the observed spectral structures are in fact normal for this experiment. It is also seen in Fig. 5 that, as the laser flux increases, the spectra tend to fill the area under the
Planck curve, indicating the trend to equilibrium. For the highest applied laser intensity the spectrally integrated flux approaches the equilibrium flux within a factor of 0.4. A better approach could be expected for more energetic laser pulses and a wall material optimized with respect to its opacity.

However, the absolute value of the radiant energy flux in the cavity is less (by a factor of $0.8-0.3$ ) than predicted. Obviously the laser falls short of the ideal source assumed in the calculations, with part of the energy being lost into non-radiative channels. Evidently, the generation of higher temperatures is not merely a question of source flux and wall opacity; the physical nature of the laser source also counts. Its role is particularly difficult to analyse owing to the complexity and non-linearity of laser-plasma interaction.

\section{Discussion and Perspectives}

There are good prospects that the laboratory generation of intense Planck radiation could evolve rapidly. Lasers are
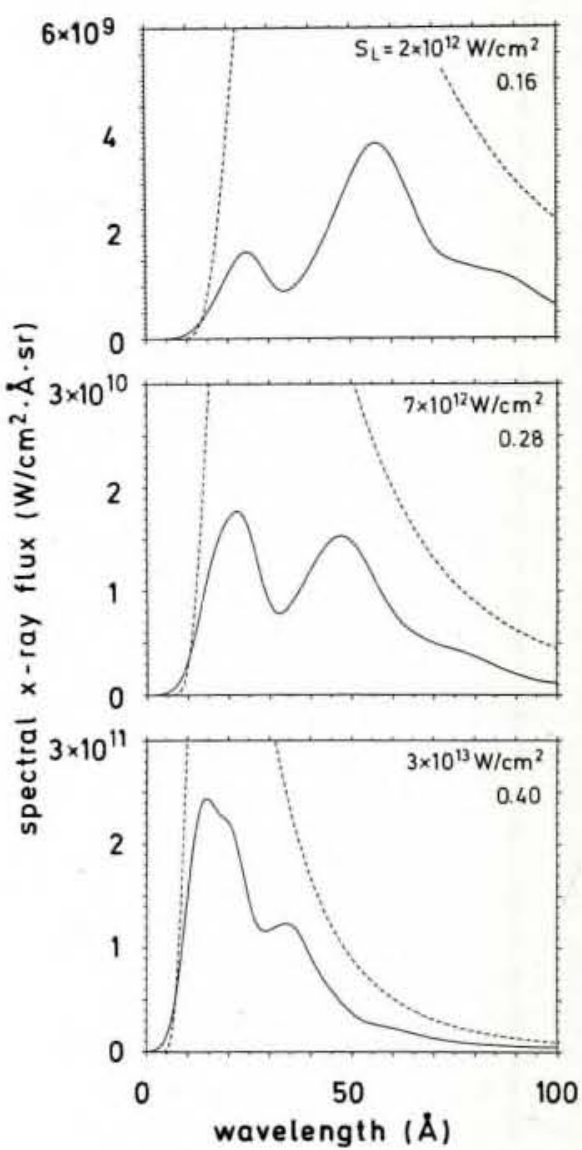

Fig. 5 - Spectra calculated for the experimental conditions of Fig. 4. With increasing laser flux $S_{L}$ the spectra approach the Planckian spectra (dashed line) corresponding to the wall temperature. The number below the $S_{L}$ value indicates the ratio of the area under the calculated to the area under the Planckian spectrum. 
by now highly developed, flexible tools, well suited to basic investigations. Continuous progress is also made in the soft $X$-ray instrumentation needed for diagnostics e.g. in the fabrication of gratings, precise surfaces for $X$-ray optics, dielectric mirrors, and detectors. Modern computers can solve the complex atomic physics problems of radiative heat transfer with adequate accuracy.

According to the present understanding of laser-plasma interaction, shortwavelength lasers seem most suitable for the generation of high-temperature radiation in a cavity.

This is not only because of their high $X$-ray conversion efficiency 10.8 for $\lambda_{L}=$ $0.26 \mu \mathrm{m}^{3}$ ) but also because of the relative absence of detrimental effects like suprathermal electron generation which dominates the laser-plasma interaction completely for the long-wavelength $\mathrm{CO}_{2}$ laser. Considerable efforts are presently made to generate high-power, shortwavelength laser radiation either by frequency conversion in crystals or by constructing excimer lasers. In the more distant future, pulsed heavy ion beams may become an alternative, because a favourable thermalization of the beam energy and a high efficiency of the generator are expected.

The scientific interest in the generation of intense Planck radiation has to do with the new possibilities to study the physics of emission and absorption of

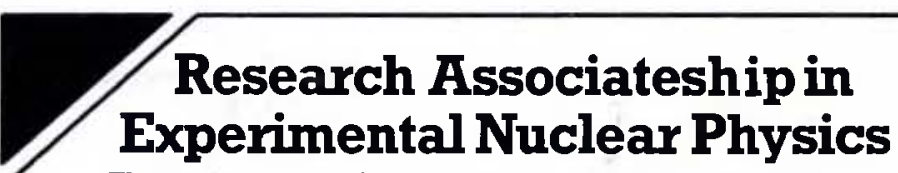

There is a vacancy for an experimental physicist to join the nuclear physics group as a Research Associate at the Daresbury Laboratory, an establishment of the Science and Engineering Research Council situated in the North Cheshire countryside.

The Group is involved in a research programme on the Nuclear Structure Facility, a large tandem accelerator which currently is operating at up to $20 \mathrm{MV}$ on terminal.

The principal areas of study are: direct reactions with light and heavy ions, production of exotic nuclei far from stability, nuclei with very high angular momentum, nuclear breakup and fragmentation and studies of isotope shifts and hyperfine structure using laserinduced resonance fluorescence. Experimental equipment includes advanced gamma-ray detector arrays, a Q3D magnetic spectrometer, and a lm scattering chamber. An isotope separator operates in either on-line or off-line modes in conjunction with an associated dilution refrigerator and a beam line for laser studies. A recoil separator has recently been brought into full operation, and a heavy-ion polarized source is in an advanced commissioning stage.

Applicants should possess a PhD degree or expect to obtain one during 1986.

An appointment will be made at a salary between $£ 9321$ and $£ 12,488$ per annum depending on age, ability and experience. The post is available for a fixed term of three years and is superannuable.

CLOSING DATE: 28 th November, 1986

For further information please write or phone Dr J. Lilley on Warrington (0925) 603558.

Application forms may be obtained from and should be retumed quoting reference $\mathrm{DL} / 970$ to: The Personnel Officer, Science and Engineering Research Council, Daresbury Laboratory, Daresbury, Warrington, Cheshire WA4 4AD, England. Tel: (0925) 603467.

\section{KERNFORSCHUNGSANLAGE JÜLICH GmbH}

The KFA is a large national research centre funded by the Federal Republic of Germany and the State of North Rhine-Westphalia.

\section{We are seeking}

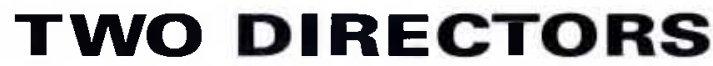

\section{jointly to lead our future alnstitute for Thin Film and Ion Technology".}

This Institute will support the research and development program of the KFA on the fundamentals of information technology by its own research contributions as well as by making available and developing a wide range of modern techniques for the deposition and characterization of thin films or film systems. By opening its facilities to universities and to industry, the Institute is also expected to become a centre for thin film preparation and analysis. A staff of 100 to 120 is envisaged for the Institute.

Topics for research and development are currently expected to include: film-systems, meeting the future requirements of information technology (multilayers of amorphous and crystalline semiconductors, magnetic and magneto-optical layers for data storage, metallization systems, layers for mechanical or chemical protection), ion and laser techniques for the manufacture, modification and analysis of films. and the development of advanced ion beam methods.

Applications for the positions are invited from scientists at universities as well as industrial and governmental laboratories. Applicants are expected to possess the capacity to lead a research establishment and to have outstanding knowledge and experience in the above fields. The candidates must be ready and willing to cooperate with industry, universities and also other institutes at the KFA.

It is planned that the directors receive tenure, as full professors at universities in North Rhine-Westphalia. The salary will conform to the C-4 scale of the German civil service. The status of a senior lecturer at a university («Habilitation") or an equivalent scientific qualification is required.

Applications accompanied by a curriculum vitae, a list of publications and a short description of previous scientific activities should be submitted by 31 October, 1986 to 


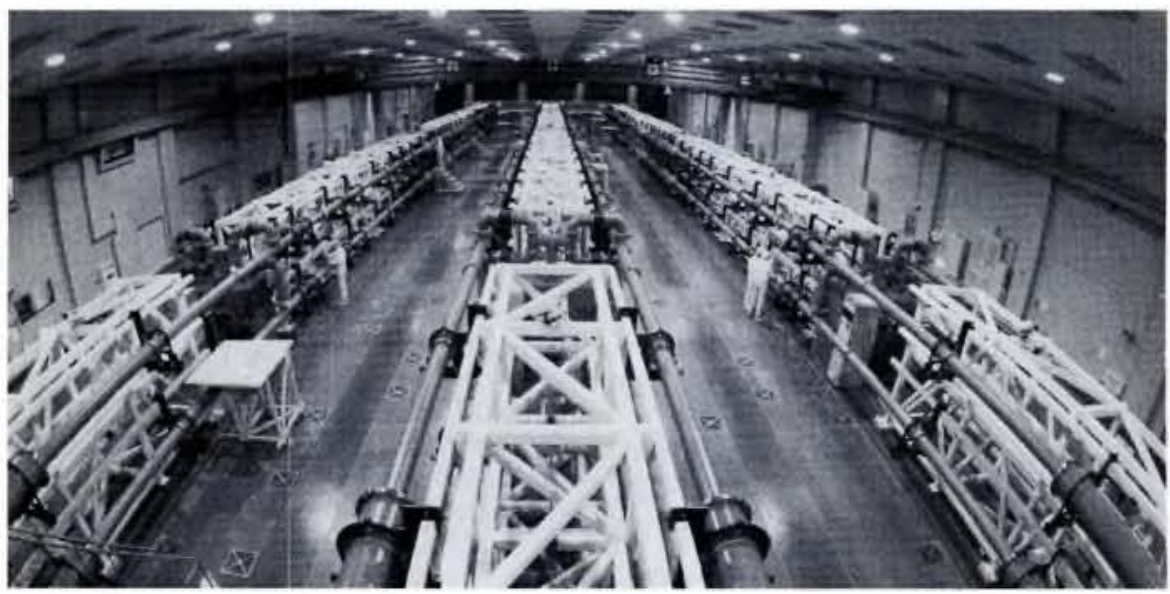

Fig. 6 - The Gekko-12 beam glass laser at the Institute for Laser Engineering, Osaka University.

radiation at high temperatures, to perform experiments in the field of radiation hydrodynamics in the laboratory and to generate in a controlled manner very high pressures by exposing a specimen to the very uniform and intense radiation in a cavity. "Astrophysics in the laboratory" is a good characterization of the direction of this new field.

The most ambitious technical application is the production of electric power by inertial confinement fusion. The principle is to implode and ignite a fusion capsule by exposing it to the intense radiation in a cavity. A large laser built for fusion research at Osaka University ${ }^{4}$ ) is shown in Fig. 6. However, even if ignition could be demonstrated in the next decade, the need to construct power sources with adequate efficiency would probably postpone economic power production into the next century. Unfortunately the research programmes in several countries are secret being primarily directed towards more immediate potential military applications. Inertial confinement fuslon is thus another example of how the results of modern science can serve or harm mankind.
This work was supported in part by the Commission of the European Communities in the framework of the Association Euratom/JPP.

\section{REFERENCES}

1. Zel'dovich Ya.B. and Raizer Yu.P., Physics of Shock Waves and High-temperature Hydrodynamic Phenomena (Academic Press, New York) 1966.

2. Pakula R. and Sigel R., Z. Naturforsch. 41a (1986) 463 and Phys. Fluids 28 (1985) 232 (see also ibid. 29 (1986) 1340).

3. Laser Program Annual Report 84, Lawrence Livermore National Laboratory, Report UCRL-50021-84 (June 1985), available from NTIS.

4. Yamanaka C. et al., in Plasma Physics and Controlled Nuclear Fusion Research 1984, Vol. 3, Nuclear Fusion Supplement 1985, p. 3: Okada K. et al., J. Appl. Phys. 59 (1986) 2332.

5. Tsakiris G.D. et al., Europhys. Lett. 2 (1986) 213. Herrmann P. et al., Z. Naturforsch. 41a(1985) 767; Földes I.B. et al., Europhys Lett. 2 (1986) 221. Eidmann K. et al., Laser and Particle Beams 4 (1986) 521. 6 . To be presented at the 11th International Conference on Plasma Physics and Controlled Nuclear Fusion Research, Kyoto, Japan, 13-20 Nov. 1986.

\section{EPS PUBLICATIONS}

The following are the subscription rates or prices for publications of the Society in 1987.

Europhysics News

10 issues

Sw.Fr. 90.-

(free to IOMs and members

of subscribing societies)

Europhysics Letters

24 issues (vols. III \& IV) Sw.Fr. 595.-

IOMs

Sw.Fr. 60. -

plus mailing contribution Sw.Fr. 10.-

Europhysics Conference Abstracts

Series 11 (8-10 titles) Sw.Fr. 330.-

Previous subscribers Sw.Fr. 290.-

European Journal of Physics
4 issues (vol. 8)
E 65 . - or $\$ 110$.

IOMs

Sw.Fr. 55.

Seminar on International

Research Facilities

IOMs

Sw.Fr. 65. -

Sw.Fr. 40.-

Still Available

Formulae and Methods in Experimental Data Evaluation ( 3 vols.) Sw.Fr. 50. IOMs

Sw.Fr. 39.-

Proceedings of the:

International Europhysics Conference on High Energy Physics, Bari,

July 1985

Sw.Fr. 90.-

European Conferences on Controlled

Fusion and Plasma Physics:

10th Conf. Moscow,

September 1981

Sw.Fr. 225.-

11th Conf. Aachen,

September 1983

12th Conf. Budapest,

September 1985

Sw.Fr. 175. -

13th European Conf. on Controlled

Fusion and Plasma Heating,

Schliersee, April $1986 \quad$ Sw.Fr. 175.

Copies are available from the EPS Secretariat in Geneva.

\section{EPS STAFF}

On 1 September 1986 Andrew Fernandes joined the staff of the EPS Secretariat in Geneva as Assistant Executive Secretary as support for the Executive Secretary, Gero Thomas.

The new voice answering the telephone belongs to Christa Zurlinden.
EPS Divisions, Sections and Group

Astronomy and Astrophysics Division Solar Physics Section

Atomic and Molecular Physics Division Atomic Spectroscopy Section Chemical Physics Electronic and Atomic Collisions Molecular Physics Computational Physics Group

Condensed Matter Division Liquids Section

Low Temperature Physics Section

Macromolecular Physics

Magnetism

Metal Physics

Semiconductors and Ins

Surfaces and Interfaces
High Energy \& Particle Physics Division

High Energy \& Particle Physics Division
Interdiv. Group on Exptl. Phys. Control Systems

Interdiv. Group on Physics for Development

Nuclear Physics Division

Optics Division

Plasma Physics Division

Quantum Electronics Division
Europhysics News is the official journal of the European Physical Society which comprises 29 National Socie ties, Academies and Group, about 4000 Individua Members and 70 Associate Members. Governing bo dies of EPS are the General Meeting, Council and an elected Executive Committee responsible for detailed policy. EPS promotes the collaboration of physicists policy. EPS promotes the callaboration of physicists
throughout Europe, organising and harmonising confethroughout Europe, organising and harmonising confe encouraging physics applications, awarding scholarships to sponsored schools in Erice. EPS publishes in addition to Europhys. News, Europhysics Letters lin partnership with national societies). European Journa partnership with national societies), European Journal of Physics (in partnership with The UK Inst. of Phys.), Members receive Europhys. News free of charge (price to insts.: Sw.Fr. 90-/a), Europhys. Lett. at Sw.Fr. 55.-/a (insts. 550.-1, rebates on many other publications and on conference fees. Annual EPS membership fee for Individual Members belonging to an EPS member society is: Sw.Fr. 44.-; independent members: Sw.Fr. 132 . members of a Collaborating Society: Sw.Fr. 55.- (\$ 26)

\section{Editor: E.N. Shaw}

Editorial Board

K. Appert, A. Baratoff, F. James,

M. Mayor, J. Muller, M. Lehmann

Edhorial and Advertising Office at the EPS Secretariat

Address: EUROPEAN PHYSICAL SOCIETY P. O. Box 69 , CH-1213 Petit-Lancy 2 Switzerland

Telephone: Geneva (22) 931130 Telex : 428024 eps ch Cables: europhys genève

Printed by: Pfirter frères sa CH-1213 Petit-Lancy/Switzerland 